
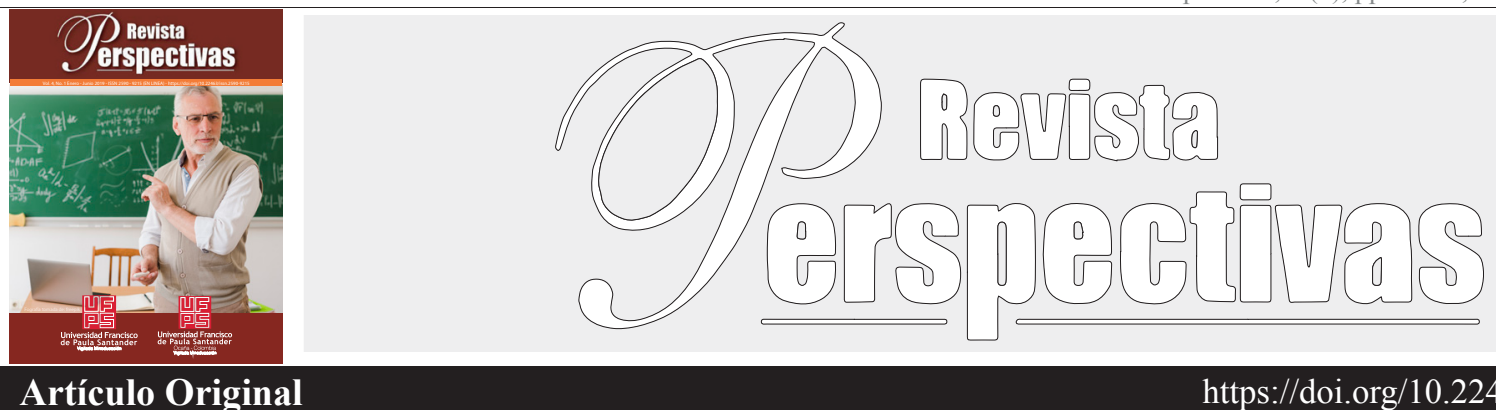

Artículo Original

https://doi.org/10.22463/25909215.1758

\title{
Ingeniería didáctica como recurso metodológico para el aprendizaje de los conceptos de límite y continuidad
}

Didactic engineering as a methodological resource for learning the concepts of limit and continuity

A engenharia didática como recurso metodológico para a aprendizagem dos conceitos de limite e continuidade

Jhoana Alexandra Trujillo-Castro ${ }^{\mathrm{a}^{*}}$, Charen Lisset Vera-Gutiérrez ${ }^{\mathrm{b}}$, Dilan Fabian Saraza-Sosa ${ }^{\mathrm{c}}$

$a^{*}$ Estudiante de pregrado en Licenciatura en Matemáticas, jhoanaalexandratc@ufps.edu.co, ORCID 0000-0001-5426-2311, Universidad Francisco de Paula Santander, Cúcuta, Colombia

${ }^{b}$ Estudiante de pregrado en Licenciatura en Matemáticas, charenlissetvg@ufps.edu.co, ORCID 0000-0002-6400-0275, Universidad Francisco de Paula Santander, Cúcuta, Colombia.

${ }^{c}$ Estudiante de pregrado en Licenciatura en Matemáticas, dilanfabianss@ufps.edu.co, ORCID 0000-0001-7469-9748, Universidad Francisco de Paula Santander, Cúcuta, Colombia.

Cómo citar: Trujillo, J. A., Vera, C. L. \& Sosa, D. F. (2019). Ingeniería didáctica como recurso metodológico para el aprendizaje de los conceptos de límite y continuidad. Perspectivas, 4(1), 39-47.

Recibido: Agosto 20, 2018; Aceptado: Noviembre 01, 2018

\begin{tabular}{ll}
\hline RESUMEN \\
\hline Palabras Clave: & $\begin{array}{l}\text { El entendimiento y comprensión de los conceptos matemáticos, específicamente de aquellos que se orientan en la educación } \\
\text { media y superior, como es el caso del límite y continuidad, es considerado uno de los mayores retos educativos para los }\end{array}$ \\
Aprendizaje & estudiantes de hoy en día, quienes tienen una concepción muy limitada acerca del concepto, tras ser vista solamente desde \\
Continuidad & su representación algebraica y lenguaje abstracto. Siendo así, el siguiente artículo de investigación tiene como objetivo \\
Ingeniería didáctica & principal responder a esa necesidad educativa, por medio de un diseño de secuencias didácticas sobre el concepto de límite \\
Límite & y continuidad, en el que está comprendido los cuatro tipos de representación matemática (numérico, algebraico, gráfico y \\
Secuencias didácticas & comunicativo) desarrollado por Duval; el cual, a su vez, se estructuro con base en unos objetivos específicos, fundamentados \\
& en la metodología de la ingeniería didáctica. De este modo, se valoró la coherencia lógica en la comprensión del concepto, \\
& que estudiantes de Licenciatura en Matemáticas de la Universidad Francisco de Paula Santander manifestaban durante el \\
desarrollo de las actividades, además de destacar las falencias y fortalezas en la construcción del mismo.
\end{tabular}

\section{ABSTRACT}

Keywords:

The understanding and comprehension of mathematical concepts, specifically those that are oriented in secondary and higher education, as is the case of limit and continuity, is considered one of the greatest educational challenges for today's students, who have a very limited conception about the concept, after being seen only from its algebraic representation and abstract

Apprenticeship

Continuity language. Thus, the following research article has as its main objective to respond to this educational need, by means of a design

Didactic Engineering

Limit of didactic sequences on the concept of limit and continuity, which includes the four types of mathematical representation (numerical, algebraic, graphic and communicative) developed by Duval; which, in turn, was structured based on specific objectives, based on the methodology of didactic engineering. In this way, the logical coherence in the understanding of the concept, which students of the Mathematics Degree of the Francisco de Paula Santander University showed during the development of the activities, as well as highlighting the weaknesses and strengths in the construction of the same, was valued.

\begin{tabular}{l}
\hline Palavras chave: \\
Aprendizagem \\
Continuidade \\
Engenharia Didática \\
Limite \\
Sequências didácticas
\end{tabular}

\section{RESUMO}

A compreensão e compreensão dos conceitos matemáticos, especificamente aqueles que são orientados no ensino médio e superior, como é o caso do limite e da continuidade, é considerada um dos maiores desafios educacionais para os estudantes de hoje, que têm uma concepção muito limitada sobre o conceito, após serem vistos apenas a partir de sua representação algébrica e linguagem abstrata. Assim, o artigo de pesquisa a seguir tem como principal objetivo responder a essa necessidade educacional, por meio de um desenho de sequências didáticas sobre o conceito de limite e continuidade, que inclui os quatro tipos de representação matemática (numérica, algébrica, gráfica e comunicativa) desenvolvidos por Duval; que, por sua vez, foi estruturada com base em objetivos específicos, baseados na metodologia da engenharia didática. Desta forma, foi valorizada a coerência lógica na compreensão do conceito, que os alunos do curso de Matemática da Universidade Francisco de Paula Santander demonstraram durante o desenvolvimento das atividades, além de destacar os pontos fracos e fortes na construção das mesmas. 


\section{Introducción}

La comprensión y dominio del concepto del límite y continuidad es uno de los focos centrales de estudio en la comunidad científica desde tiempos remotos, una de las razones es porque resulta ser un conocimiento necesario para la asimilación de los contenidos que abarca el cálculo diferencial e integral y la segunda razón que motiva su investigación es la dificultad existente durante su proceso de aprendizaje. Siendo así, Radillo Enríquez y González Rendón (2014) señalan que las metodologías de enseñanza dedicadas al aprendizaje de conceptos matemáticos específicamente en el del límite establecen una visión reducida del concepto, al enseñar el mismo a partir de ejercicios mecánicos que funcionan en la importancia de operaciones algebraicas. Además, Cornu, (1983) y Bouazzoui (1988) citado en (Sánchez Gómez y Contreras De La Fuente, 1998) y (Quevedo, 2018) afirman que los obstáculos de origen epistemológico son propios del concepto matemático, durante su proceso de construcción, pero, a su vez estos influyen en las dificultades de aprendizaje y concepciones erróneas.

Conforme a lo dicho, Medina (2001) señala que el estudio de las diferentes concepciones a través de la historia de cualquier concepto, en este caso, del límite, es muy importante para la comprensión del tema, en el cual se aprecian diferentes aplicaciones que se le han dado a través del tiempo; y sirve de introducción para el desarrollo del pensamiento variacional del cálculo. Además, Duval (1998) citado en (Volveras Espinosa, 2015) expresa que

"la formación de conceptos implica una coordinación de sistemas de representaciones, esta se logra articulando entre diferentes registros. Entendemos por representaciones, diferentes notaciones, ya sean gráficas, simbólicas, así como expresiones verbales. Estas representaciones se agrupan en registros. Por ejemplo, el registro gráfico o el registro numérico. Entonces un reto importante en el aprendizaje de la matemática no puede ser, solamente, la automatización de ciertas técnicas operatorias, sino que debe ser también, la coordinación de los diferentes sistemas de representación".

De acuerdo con lo anterior es importante resaltar la preocupación que manifiesta los investigadores en didáctica de la matemática en entender los obstáculos que surgen durante la enseñanza y el aprendizaje del límite, el cual es uno de los conceptos principales que se da dentro de la asignatura de cálculo diferencial y el mismo abarca temáticas sobre problemas de economía, administración, natural y social. Siendo así, se destaca a Vrancken, Gregorini, Engler, Müller y Hecklein, (2006) los cuales llevan a cabo un estudio basado en la metodología de la ingeniería didáctica y representaciones semióticas de Duval, con el objetivo de hallar las dificultades relacionadas al concepto de limite que presentan los estudiantes de educación superior, llegando a la conclusión que las dificultades presentes en el tema se debe a concepciones erróneas de los conceptos previos como en las funciones y la factorización. Por otro lado Blázquez, Ortega , Gatica y Benegas, (2006)en su objetivo de comparar el nivel de complejidad entre la concepción métrica y aproximación optima del límite a través de una revisión histórica del concepto concluyen que para los estudiantes la conceptualización del límite como aproximación óptima para explicar los teoremas es más útil, puesto que sus demostraciones son más sencillas, más intuitivas (numéricas - algebraicas) aunque carecen de formalidad como es en el caso de la apreciación métrica del concepto. Además, Sierra Vázquez, González Astudillo y López Esteban (2000) en su objetivo conocer las concepciones de los estudiantes alrededor de tema de límites, concluye que existe una gran diversidad y esta es consecuencia de la evolución epistemológica del concepto a nivel geométrico, aritmético, algebraico, funcional y topológico; en un segundo lugar encontró tras realizar un cuestionario, que los estudiantes presentan dificultades en las representaciones semióticas del concepto siguiendo un nivel de complejidad que trasciende en las representaciones tabular, gráfica y algebraica. Las conclusiones de cada una de esta investigación se fortalecen en las medidas en que trabajos como los realizados por (Morales et al., 2013 y Sierra et al., 2000) recalcan las mismas dificultades y errores en cuanto al concepto de límite.

Como consecuencia del panorama descrito consideramos la importancia de generar un material didáctico que fortalezca la comprensión del concepto desde sus diferentes perspectivas y representaciones, como lo manifiesta Sierpinska (1990) citado en (Volveras Espinosa, 2015) Quien plantea que para haiga un aprendizaje significativo este debe ser modelizado a partir de secuencias de comprensión y superación de errores. De esta manera, La teoría que sustenta este trabajo de investigación es la Teoría de Situaciones Didácticas de Duval y la metodología a usar es la Ingeniería Didáctica; ya que la articulación de ambas considera el tener en cuenta aspectos importantes para el cumplimiento del objetivo y simultáneamente para la construcción del concepto.

\section{Materiales y métodos}

Como se había descrito anteriormente la metodología en la cual se basa la investigación y a la que más se ajusta es la denominada ingeniería didáctica, la cual se centra en organizar un análisis general de conceptos que necesitan ser estudiados desde una perspectiva científica con la finalidad de responder a necesidades educativas; como lo expresa Douady (1995) citado en Montiel y Navarro (2015) una ingeniera didáctica, es un conjunto de secuencias de clase diseñadas, organizadas y articuladas coherentemente por un "profesor-ingeniero", para lograr el aprendizaje de cierto conocimiento en un grupo de alumnos específico. Siguiendo este orden de ideas la ID está conformada por cuatro fases: 
1) Fase 1 Análisis preliminar; la cual hace referencia en primer lugar al análisis epistemológico de los contenidos contemplados en la enseñanza y de manera simultánea al análisis de las metodologías de enseñanza y concepciones de los estudiantes.

2) Fase 2 Concepción y análisis a priori de la ingeniera didáctica; la cual establece el análisis de las variables que intervienen en la investigación; en este caso todas las variables relacionadas con la enseñanza y aprendizaje del concepto de limite.

3) Fase 3 Experimentación; relacionado con el diseño del material didáctico.

4) Fase 4 Análisis a posteriori y evaluación; que hace referencia al análisis de resultados.

Bajo estas consideraciones, la presente investigación expone la realización de las fases en el siguiente orden de ideas. De acuerdo con la primera fase se presenta un análisis epistemológico del concepto, seguido de un bosquejo general acerca de la presentación institucional que ha tenido el tema de límites por medio de una de las herramientas más utilizadas por los docentes que es el libro, para posteriormente finalizar con una descripción breve sobre las dificultades generales que presentan los estudiantes según autores reconocidos como Cornu y Sierpinska. En segundo lugar, en el análisis a priori se llevó a cabo un rastreo de tipo documental derivado de un estado del arte alrededor del concepto de límite, el cual tenía como objetivo identificar los tipos de investigación, las falencias y potencialidades conforme al concepto de límites, además, se tuvo en cuenta otros trabajos investigativos procedentes de diferentes bases de datos; en la tercera fase de experimentación se orienta la realización de una secuencias didácticas previamente desarrolladas bajo la consideración de los resultados obtenidos en los dos primeras fases. Y para finalizar, la cuarta fase consiste en un análisis de resultados obtenidos mediante una evaluación. La población sujeta a este trabajo de investigación fueron 27 docentes en formación que cursaban la asignatura de Calculo Diferencial, en el programa académico de licenciatura en matemáticas de la Universidad Francisco de Paula Santander (UFPS) durante el primer semestre del año 2018.

\section{Análisis}

\section{Análisis preliminar}

\section{Análisis epistemológico del concepto.}

La evolución del concepto de limite se presenta como una secuencia de modificaciones que son el resultado de su compresión desde diferentes perspectivas o categorías (geométrica, numérica, funcional, algebraica, topológica), siendo así, a continuación, se presenta una descripción histórica de las diversas concepciones a las que se orientó el concepto de límite y que fueron necesarias para la construcción formal del mismo, teniendo como base los trabajos realizados por Espíritu Montiel y Navarro (2015), Quevedo (2018); Volveras Espinosa (2015) y Medina (2001). Ver tabla 1

Tabla 1. Origen epistemológico del concepto de limite

\begin{tabular}{|c|c|}
\hline $\begin{array}{c}\text { Desde el año } \\
400 \text { A.C. hasta } \\
\text { la primera } \\
\text { mitad del Siglo } \\
\text { XVIII }\end{array}$ & $\begin{array}{l}\text { durante este periodo se evidencia la idea intuitiva sobre el concepto de límite, por } \\
\text { parte de los griegos entre los años } 400 \text { A.C y } 300 \text { A.C., el cual lo consideran como } \\
\text { un proceso implícito para resolver problemas de velocidad, tangentes a curvas y } \\
\text { cálculo de áreas. Sumado a esto Newton (1704) y Leibniz (1646-1716) se aproximan } \\
\text { al concepto en un mismo carácter geométrico, newton por su parte reconoce que "El } \\
\text { límite es una cantidad (Cociente) a la cual una razón de cantidades en movimiento se } \\
\text { aproxima continuamente, más que cualquier diferencia dada y no puede alcanzarla o } \\
\text { sobrepasarla antes que las cantidades hayan decrecido indefinidamente" (Medina M., } \\
\text { 2001). Leibniz por su parte se dio cuenta en la teoría de cálculo diferencial que "la } \\
\text { pendiente de la tangente a una curva depende de la razón entre las diferencias de las } \\
\text { ordenadas y de las abscisas, cuando estas diferencias se hacen infinitamente } \\
\text { pequeñas" (Espíritu Montiel \& Navarro, 2015). Aquí es importante resaltar que antes } \\
\text { de los aportes de newton se encuentran los hallazgos realizados por Johannes Kepler } \\
\text { (1571-1630), Bonaventura Cavalieri (1598-1647) y Pierre de Fermat (1601-1665) los } \\
\text { cuales, tras trabajar con figuras geométricas para hallar el área, el volumen o los } \\
\text { extremos de una curvan, introducen el concepto del análisis infinitesimal que } \\
\text { posteriormente fue debatido por diferentes matemáticos. además de lo anterior según } \\
\text { Bouazzaoui (1988), Cornu (1983) y Deledicq (1994) este primer avance histórico } \\
\text { hace referencias a la primera concepción que obtuvo del concepto de limite el } \\
\text { geométrico. }\end{array}$ \\
\hline & $\begin{array}{l}\text { Con la necesidad de operar con un mayor número de funciones Euler (1707-1743) } \\
\text { realiza la introducción a procesos infinitos dando la noción de funciones continuas a } \\
\text { partir de operaciones elementales. dados estas condiciones la primera definición que } \\
\text { acerca a la formal del límite surge por parte de D'Alembert en (1717-1783) en el tomo } \\
\text { IX de la Encyclopédie, argumentando que el límite de una cantidad se da cuando esta } \\
\text { se aproxima tanto al límite que en el momento de establecer sus diferencias este será } \\
\text { absolutamente inasignable, en contraste con D'Alembert; Lagrange (1736-1813) } \\
\text { consiguió que los limites no eran necesarios, tras realizar un trabajo sobre funciones } \\
\text { en serie de potencias. según Bouazzaoui (1988) y Cornu (1983) y Deledicq (1994) en } \\
\text { esta segunda etapa se destacan el desarrollo de concepciones numéricos que se } \\
\text { demuestra en el uso de sucesiones para las variables dependiente e independiente de } \\
\text { una función. }\end{array}$ \\
\hline $\begin{array}{c}\text { Finales del siglo } \\
\text { XVIII y } \\
\text { comienzos del } \\
\text { siglo XIX }\end{array}$ & $\begin{array}{l}\text { Esta etapa se caracteriza por la intención de muchos matemáticos de establecer una } \\
\text { definición formal del concepto, por ejemplo, Cauchy presenta una definición muy } \\
\text { similar a la de D’Alembert, pero con un carácter aritmético, además Weierstrass } \\
\text { (1815-1897) da una visión métrica del concepto integrándolo al de función con } \\
\text { valores como él } \varepsilon \text { y } \delta \text { y el uso de los cuantificadores. en esta parte de la historia se } \\
\text { destaca la disputa entre D'Alambert y Cauchy sobre si el límite es alcanzado o no. } \\
\text { Además de lo anterior según El Bouazzaoui (1988), Cornu (1983) y Deledicq (1994) } \\
\text { esta última etapa hace referencia a concepciones métricas y topológicas }\end{array}$ \\
\hline
\end{tabular}

Fuente: Espíritu Montiel y Navarro (2015); Quevedo (2018); Volveras Espinosa (2015); Medina (2001).

Análisis del desarrollo del concepto de límite en libros institucionales.

Con este tipo de análisis lo que se pretende es conocer cómo se desarrolla el concepto en un nivel institucional, tomando como referencia los libros dado que es la herramienta más utilizada y cercana con la que cuenta el profesor para la enseñanza y el estudiante para su aprendizaje del concepto de límite en particular. siendo así, para este análisis se tuvo en cuenta el estudio realizado por (Artigue, Douady y Moreno, 1995). Ver tabla 2 
Tabla 2. Desarrollo del concepto de límite en libros institucionales.

\begin{tabular}{|c|c|}
\hline $\begin{array}{l}\text { Primer periodo } \\
(1940-1967)\end{array}$ & $\begin{array}{l}\text { En este periodo el concepto de límites fue presentado en un lenguaje de sucesiones, } \\
\text { es decir hacían referencia al hallazgo de límites en una sucesión de valores, donde } \\
\text { se verificaba la existencia del mismo a partir de la diferencia de uno de esos valores } \\
\text { y el límite; seguido de este primer modelo conceptual el cual se caracterizó por } \\
\text { poseer un carácter algebraico y numérico, surge la introduccíon del límite a partir } \\
\text { de funciones que aportan un carácter geométrico. Por ejemplo } \\
\text { El límite de la función f(x) cuando x tiende a es el número b, si se } \\
\text { verifica que los valores de la función f(x) se aproximan tanto como } \\
\text { queramos a b, tomando los valores de la variable x convenientemente } \\
\text { próximos a a (no se hace ninguna hipótesis sobre el valor de la función en } \\
\text { el punto a) (Artigue, Douady y Moreno, 1995, p.466). }\end{array}$ \\
\hline $\begin{array}{l}\text { Segundo periodo } \\
(1967-1975)\end{array}$ & $\begin{array}{l}\text { En los cuales se aplica el concepto integrándolo a un carácter métrico y topológico } \\
\text { ajustado a representaciones graficas como el diagrama de ven y a representaciones } \\
\text { simbólicas con el uso de cuantificadores; este periodo se caracteriza por presentar } \\
\text { el concepto en un nivel más complejo en donde el papel didáctico del mismo queda } \\
\text { reducido a la a resolución de ejercicios mecánicos y demostraciones sin articulación } \\
\text { contextual. Por ejemplo, } \\
\text { Se dice que la función } f(\mathrm{x}) \text { tiende hacia L cuando x tiende hacia }+\infty \text { (o } \\
\text { hacia - } \infty \text { ), si para todo } \varepsilon>0 \text { existe un real positivo } \mathrm{A} \text {, tal que }|\mathrm{x}|>\mathrm{A} \\
\text { implica } \\
|f(\mathrm{x})-\mathrm{L}|<\varepsilon ; \forall \varepsilon>0, \exists \mathrm{A}>0 \text { tal que }|\mathrm{x}|>\mathrm{A} \Rightarrow \mid f(\mathrm{x}) \text { )-b } \mid<\varepsilon \text {. (Artigue, } \\
\text { Douady y Moreno, 1995. p.469). }\end{array}$ \\
\hline $\begin{array}{l}\text { Tercer periodo } \\
(1975-1995)\end{array}$ & $\begin{array}{l}\text { En este áltimo periodo se introdujo el concepto a partir de conjeturas dadas por el } \\
\text { cumplimiento del limite bilateral, el limite infinito y el limite en el infinito, los } \\
\text { cuales se debían estudiar en las diferentes representaciones. }\end{array}$ \\
\hline
\end{tabular}

Fuente: (Artigue, Douady y Moreno, 1995)

\section{Análisis de las dificultades o errores epistemológicos según Cornu (1991) y Sierpinska (1985)}

En este último paso del análisis preliminar lo que se pretende es realizar un descripción sobre las dificultades en el aprendizaje de limite que está directamente relacionado con la evaluación epistemológica del concepto tomando como referencias los trabajos realizados por Quevedo (2018) y Sánchez Gómez y Contreras De La Fuente (1998), siendo así, en un primer lugar se halla que la concepción de limites por medios geométricos genera la facilidad de desarrollar problemas de ares y volumen en los que interviene intuitivamente procesos infinitos, pero la dificultad se presenta en el momento de comprender el proceso bajo un carácter numérico o algebraico (hallando aquí la dificultad de pasar de un registro grafico a un registro numérico); en un segundo lugar están los obstáculos referente a lo infinitamente pequeño o infinitamente grande en la noción limite funcional, el cual hace creer que ambas variables toman el valor de infinito) y por ultimo está el obstáculo relacionado con el alcance o no del límite, que en si son dificultades relacionadas con aspectos geométricos y topológicos al creer que $\varepsilon$ y $\delta$ pueden representar magnitudes o variables.

\section{Análisis a priori}

En el análisis a priori se tuvo en cuenta un rastreo de tipo documental derivado de un estado del arte alrededor del concepto de límite, además, de otros trabajos investigativos procedentes de diferentes bases de datos; los cuales dan información acerca de las diferentes metodologías que se han aplicado para la enseñanza del concepto y las falencias de los estudiantes durante su proceso de aprendizaje.
Tabla 3. Revisión de antecedentes.

\begin{tabular}{|c|c|}
\hline Autores & Descripción \\
\hline $\begin{array}{c}\text { Espíritu } \\
\text { Montiel y } \\
\text { Navarro (2015) }\end{array}$ & $\begin{array}{l}\text { Expresa el objetivo de analizar la manera en que estudiantes entre } 17 \text { y } 18 \text { años de } \\
\text { edad realizan cálculos de límites indeterminado utilizando un carácter algebraico. De } \\
\text { esta manera, para el alcance del mismo se basaron en la metodología de ingeniería } \\
\text { didáctica y la teoría de secuencias didácticas desarrollada por Guy Brousseau. Siendo } \\
\text { así, en los resultados se consigue que los estudiantes consideran el limite como una } \\
\text { sustitución de valores en vez de una aproximación optima, dichos errores fueron } \\
\text { detectados en representaciones gráficas, tabular y algebraicas; que posteriormente } \\
\text { fueron modeladas en un menor grado tras la secuencia didáctica. }\end{array}$ \\
\hline Quevedo (2018) & $\begin{array}{l}\text { Afirman que el proceso en que evoluciona el concepto de limite pasa inicialmente } \\
\text { por un carácter geométrico, seguido del el metafísico dado por la expresión } \\
\text { infinitesimal del concepto para finalizar en uno aritmético o algebraico; está } \\
\text { secuencia de concepciones está ligada con las dificultades de los estudiantes, por } \\
\text { ejemplo, en comprender que los limites se pueden trabajar desde diferentes } \\
\text { perspectivas y en un carácter infinitesimal; además de lo anterior también se llegó a } \\
\text { la conclusión que algunos errores que manifiestan los estudiantes en el momento de } \\
\text { realizar cálculos de limites se debe a concepción erróneas de preconceptos en el } \\
\text { desarrollo del algebra y la factorización. }\end{array}$ \\
\hline $\begin{array}{l}\text { Bustos, } \\
\text { Naranjo, Pisco, } \\
\text { Torrez y } \\
\text { Romero }(2016)\end{array}$ & $\begin{array}{l}\text { Los estudiantes mejoran la interpretación del límite a partir del estudio de los limites } \\
\text { laterales bajo una representación gráfica y tabular, manifestando preferencias por } \\
\text { analizar los límites con la ayuda de las TIC; pero presentan errores en cuanto a la } \\
\text { puesta de cantidad pequeñas que se aproximen al límite dado que lo expresan en } \\
\text { números enteros, además existe la dificultad para la interpretación simbólica del } \\
\text { concepto. }\end{array}$ \\
\hline $\begin{array}{c}\text { Volveras } \\
\text { Espinosa (2015) }\end{array}$ & $\begin{array}{l}\text { Trajo consigo una propuesta de enseñanza del concepto de límite de funciones para } \\
\text { el grado } 11 \text {, desarrollando una metodología didáctica que incluye el uso del software } \\
\text { educativo Geogebra; llegando a la conclusión que el estudiante presenta una mejoría } \\
\text { contextual del concepto con la ayuda de herramientas tecnológica, pero al igual se } \\
\text { debe orientar este proceso ya que presentan errores de conceptualización. }\end{array}$ \\
\hline $\begin{array}{l}\text { Radillo } \\
\text { Enríquez y } \\
\text { González } \\
\text { Rendón (2014) }\end{array}$ & $\begin{array}{l}\text { Trazo como objetivo diseñar una propuesta didáctica que le permitirá a los } \\
\text { estudiantes establecer una articulación entre las diferentes representaciones y } \\
\text { visualizarlas por medio de un recursos tecnológico, de esta manera, se concluye que } \\
\text { los estudiantes presentan dificultades para hallar los límites en funciones definidas a } \\
\text { trozos además le resulta complejo la interpretación de gráficas y la comunicación de } \\
\text { resultados debido a la simbología del concepto; pero aun así, se le agrega el aporte } \\
\text { de desarrollo conceptual por parte de las herramientas tecnológicas como el } \\
\text { Geogebra. }\end{array}$ \\
\hline $\begin{array}{l}\text { Sierra Vázquez, } \\
\text { González } \\
\text { Astudillo y } \\
\text { López Esteban, } \\
\text { (2000) }\end{array}$ & $\begin{array}{l}\text { Fijo como objetivo el analizar las concepciones de los estudiantes alrededor del } \\
\text { concepto de límites y continuidad con la finalidad de verificar si existe una relación } \\
\text { con el desarrollo del concepto a través de la historia. De esta manera se concluye que } \\
\text { la mayoría de los estudiantes tienen nociones intuitivas de ambos conceptos, en el } \\
\text { caso del límite se observa concepciones acerca de la aproximación en limites laterales } \\
\text { y con respecto a la continuidad se concibe concepciones sobre la función definida en } \\
\text { un punto y su infinita prolongación sin corte alguno. además de lo anterior se } \\
\text { concluye que las concepciones de los estudiantes están estrictamente relacionadas } \\
\text { con las controversias que han tenido los matemáticos para lograr la formalización del } \\
\text { mismo. }\end{array}$ \\
\hline $\begin{array}{l}\text { Navarro } \\
\text { Sandoval, } \\
\text { Romero } \\
\text { Valencia y } \\
\text { Miranda Nava, } \\
\text { (2012) }\end{array}$ & $\begin{array}{l}\text { Rescata la importancia de indagar acerca de la presentación de concepciones del } \\
\text { concepto de limite en los diferentes planes de estudio además de los obstáculos } \\
\text { epistemológicos, cognitivos y didácticos sobre el concepto, para posteriormente } \\
\text { llevar a cabo un buen diseño de secuencia didácticas que tenga en cuenta los aspectos } \\
\text { anteriores en las diferentes representaciones semióticas propuestas por Duval. }\end{array}$ \\
\hline $\begin{array}{c}\text { Buendía Abalos } \\
\text { y Molfino Vigo, } \\
(2010)\end{array}$ & $\begin{array}{l}\text { El proceso de esta investigación se enfocó en dos aspectos: el desarrollo social- } \\
\text { histórico del concepto del límite en la comunidad matemática y la situación actual en } \\
\text { el ámbito escolar. En el primer aspecto se concluye que el constante cambio para la } \\
\text { institucionalización del concepto del límite se debe a la búsqueda de una } \\
\text { fundamentación donde se aplique el concepto para resolver problemas de cálculo } \\
\text { diferencial e integral, su difusión al público, su generalización para nuevos conceptos } \\
\text { y su extensión a nuevos contextos de aplicación. En el segundo aspecto se puede } \\
\text { concluir que la mayoría de los docentes en matemáticas enseñan y hacen mucho } \\
\text { énfasis en este tema, porque es la base para la compresión de otros temas como la } \\
\text { derivación y continuidad; respondiendo más que todo a una necesidad de la } \\
\text { comunidad científica que a la propia necesidad de los estudiantes. }\end{array}$ \\
\hline $\begin{array}{l}\text { Contreras de la } \\
\text { Fuente, García } \\
\text { Armenteros y } \\
\text { Font Moll, } \\
\text { (2012) }\end{array}$ & $\begin{array}{l}\text { El estudio realizado sobre la estructura y funcionamiento de una clase de matemáticas } \\
\text { en la que se enseña el concepto de límite de una función, según las trayectorias } \\
\text { epistémicas e instruccionales nos señala que: la clase dada por el docente tiene una } \\
\text { configuración dialógica lo que facilita un poco el proceso de enseñanza y aprendizaje. } \\
\text { El error de sentido semiótico se da más que todo por no enseñar las diferentes formas } \\
\text { de representación (grafico, numérico y algebraico) del tema. Creando en los } \\
\text { estudiantes imágenes erróneas sobre el concepto de límite de una función }\end{array}$ \\
\hline $\begin{array}{c}\text { Donmez y } \\
\text { Basturk, (2010) }\end{array}$ & $\begin{array}{l}\text { En esta investigación se concluye, que en general, la mayoría de los docentes en } \\
\text { formación tienen claro conocimiento de la variedad de métodos de enseñanza y } \\
\text { aprendizaje que existen para la explicación de cualquier tema de matemáticas, pero, } \\
\text { en el momento de realizar sus clases no las aplican, ya que solo se centran en el } \\
\text { método de preguntas y respuestas, y uno que otro se preocupa por explicar el } \\
\text { significado que tiene el tema en la realidad, por tal motivo se confirma la deficiencia } \\
\text { de viarios cursos de formación de docentes y la necesidad de reorganizar el plan de } \\
\text { estudios designando para los docentes en formación. }\end{array}$ \\
\hline $\begin{array}{c}\text { Engler, y otros, } \\
(2008)\end{array}$ & $\begin{array}{l}\text { En el aprendizaje de los limites; se sugiere que los docentes deben brindarles la } \\
\text { oportunidad a los estudiantes para argumentar sobre los conceptos, exponer sus } \\
\text { propias ideas, para dudar de lo que dice su profesor o compañero, y para aprender a } \\
\text { aprender en grupo, con la ayuda de su compañero y guía del docente, con el fin de } \\
\text { superar las dificultades presentes el concepto del límite. }\end{array}$ \\
\hline $\begin{array}{l}\text { Morales } \\
\text { Carballo, Reyes } \\
\text { García y } \\
\text { Hernández } \\
\text { Gómez (2013) }\end{array}$ & $\begin{array}{l}\text { Tras una ejecución de actividades didácticas se demuestra que la mayoría de los } \\
\text { estudiantes presentan dificultades, tales como: Justificar el límite, aplicar las } \\
\text { condiciones del concepto, determinar el límite utilizando la representación gráfica, } \\
\text { entre otros. Siendo estas las consecuencias de no formalizar el concepto de límite, y } \\
\text { tener tan solo ideas intuitivas sobre el tema. }\end{array}$ \\
\hline
\end{tabular}




\section{Resultados y discusiones}

Teniendo en cuenta el análisis epistemológico entorno a la evolución del concepto de limite y el rastreo de antecedentes investigativos relacionados con el mismo, en donde se tuvieron en cuenta los objetivos principales y los resultados que permitían reconocer las dificultades presentes en el aprendizaje. Partiendo de lo anterior se diseñaron y aplicaron secuencias didácticas utilizando diferentes representaciones semióticas (grafica, algebraica, tabular y lenguaje natural), con el fin fortalecer las falencias encontradas en la aprehensión del concepto. Finalizada la aplicación de las secuencias, se estableció una prueba para caracterizar las concepciones presentes en 29 estudiantes de Licenciatura en Matemáticas de la Universidad Francisco de Paula Santander en el I semestre del 2018.

3.1. Prueba de conocimientos: se plantearon ocho ejercicios para determinar el nivel de desempeño de los estudiantes en la compresión del concepto de límite en sus diferentes representaciones. A continuación, se muestra en la tabla 4 la representación utilizada en cada ejercicio.

Tabla 4. Representación semiótica utilizada en cada ejercicio

\begin{tabular}{cc}
\hline Ejercicio & Representación semiótica \\
\hline 1 & Lenguaje natural y grafico \\
2 & Algebraico, tabular y grafico \\
3 & Algebraico, tabular y grafico \\
4 & Lenguaje natural \\
5 & Algebraico \\
6 & Grafico \\
7 & Gráfico y algebraico \\
\hline
\end{tabular}

3.1.1. Selección de ejercicios: los estudiantes debían elegir cuatro ejercicios de los siete propuestos, de modo que pudieran escoger aquellos que creían tener mayor probabilidad de solucionar. En la Figura 1 se muestra la elección de los estudiantes.

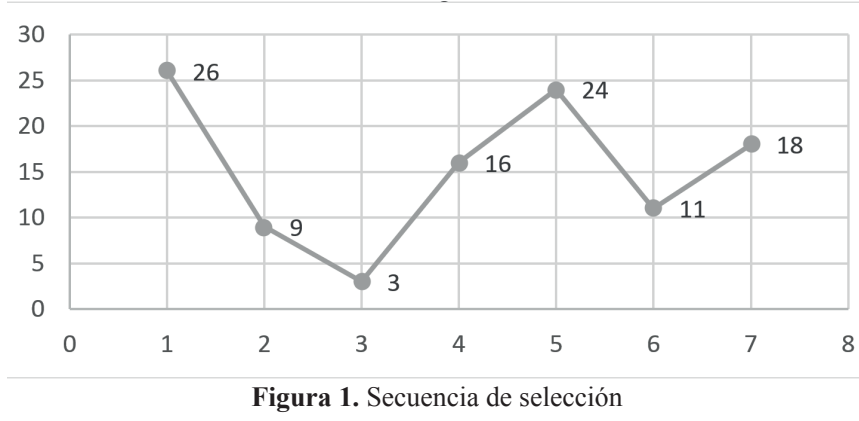

3.1.2. Niveles de desempeño obtenidos en cada ejercicio. Para el análisis de los desempeños obtenidos por los estudiantes en cada uno de los ejercicios, se tuvieron en cuenta las siguientes escalas cuantitativas presentes en la tabla 5.
Tabla 5. Escalas de desempeño

\begin{tabular}{cc}
\hline Desempeño & Valor \\
\hline Bajo & 0 a 0.42 \\
Medio & 0.43 a 0.85 \\
Alto & 0.86 a 1.25 \\
\hline
\end{tabular}

A continuación, se muestra en la tabla 6 el desempeño obtenido por los estudiantes en la prueba aplicada. Para la elaboración de tabla se tuvo en cuenta la frecuencia de selección de las preguntas y la escala de desempeño para la sistematización de la información.

Tabla 6. Resultados de la prueba

\begin{tabular}{cccc}
\hline \multirow{2}{*}{ Ejercicio } & \multicolumn{3}{c}{ Nivel de desempeño } \\
\cline { 2 - 4 } & Bajo & Medio & Alto \\
\hline 1 & $8 \%$ & $15 \%$ & $77 \%$ \\
\hline 2 & $44 \%$ & $33 \%$ & $22 \%$ \\
\hline 3 & $33 \%$ & $0 \%$ & $67 \%$ \\
\hline 4 & $13 \%$ & $88 \%$ & $0 \%$ \\
\hline 5 & $54 \%$ & $38 \%$ & $8 \%$ \\
\hline 6 & $55 \%$ & $27 \%$ & $18 \%$ \\
\hline 7 & $11 \%$ & $6 \%$ & $83 \%$ \\
\hline
\end{tabular}

3.1.3. Especificación de los ejercicios planteados en la prueba. Para la exposición de las actividades planteadas se tendrán en cuenta: objetivo de aprendizaje, comentario de la resolución de algunos estudiantes, según las dificultades y fortalezas presentadas.

El primer punto tenía como objetivo principal reconocer si los estudiantes comprendían el concepto de límite, esto les permitiría representar el concepto a través del lenguaje natural y gráfico.

1. Represente gráficamente dos situaciones, una en donde existe límite de una función en un punto cualquiera y otro, en donde dicho límite no exista, argumentado cada respuesta.

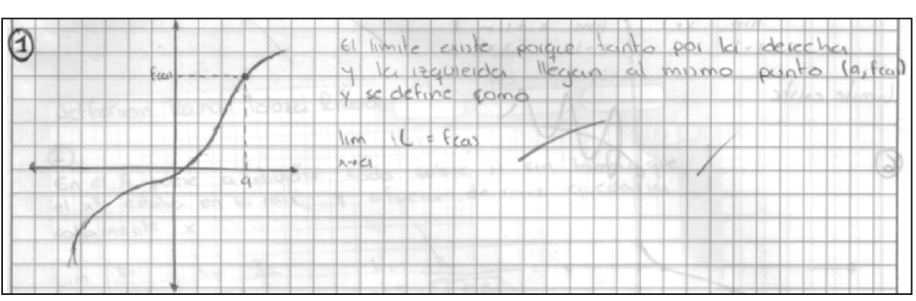

Figura 2. Resolución de ejercicio 1 - situación con limite

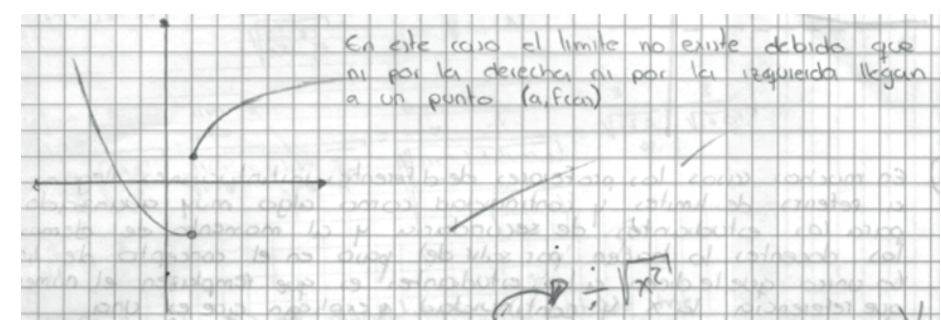

Figura 3. Resolución de ejercicio 1 - situación sin limite 
Con base en los resultados generales de la Tabla 6 se muestra que el $77 \%$ de los estudiantes tuvieron un desempeño alto en la resolución del primer punto de la prueba, por lo que se puede inferir que los educandos tienen una excelente comprensión del concepto de límite, además que tienen la capacidad de utilizar el lenguaje natural y gráfico de manera correcta, esto se puede evidenciar en las Figuras 2 y 3 mostradas anteriormente.

El segundo ejercicio tenía como objetivo identificar si los estudiantes podían hallar el límite de una función por partes, por medio de las representaciones semióticas (tabular, gráfico o algebraico), se podía hacer con un solo registro.

2. Evalué el límite de las siguientes funciones en el punto indicado.

$$
\begin{aligned}
& h(x)=\left\{\begin{array}{cr}
\sqrt[3]{x+1} & \text { si } x \leq-1 \\
\sqrt{1-x^{2}} & \text { si }-1<x<1 \\
\sqrt[3]{x-1} & \text { si } 1 \leq x
\end{array}\right. \\
& \lim _{x \rightarrow-1^{+}} h(x), \lim _{x \rightarrow-1^{-}} h(x), \lim _{x \rightarrow-1} h(x) \\
& g(x)= \begin{cases}3+x^{2} & \text { si } x<-2 \\
0 & \text { si } x=-2 \\
11-x^{2} & \text { si }-2<x\end{cases} \\
& \lim _{x \rightarrow-2^{+}} g(x), \lim _{x \rightarrow-2^{-}} g(x), \lim _{x \rightarrow-2} g(x)
\end{aligned}
$$

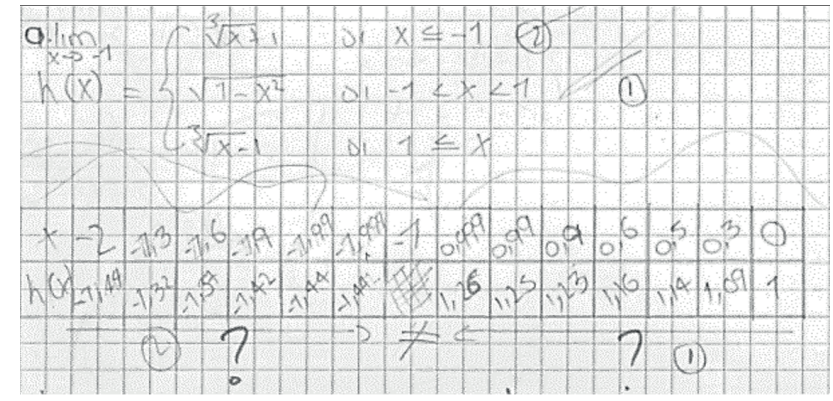

Figura 4. Resolución de ejercicio 2a

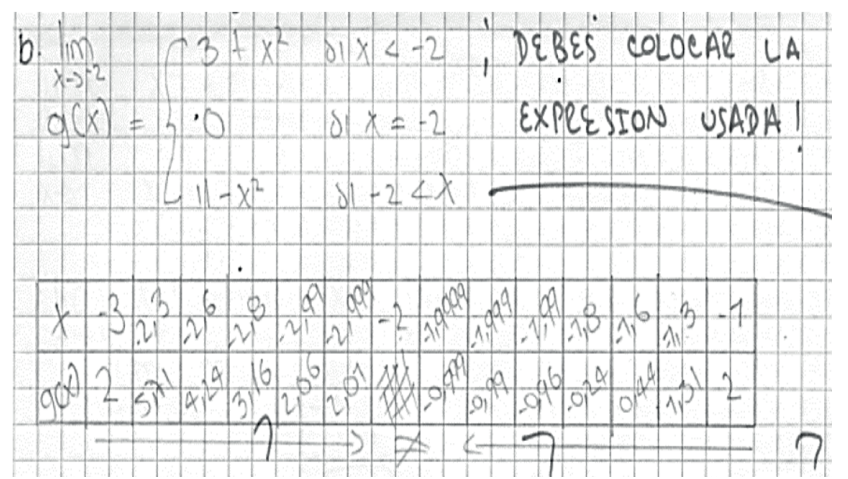

Figura 5. Resolución de ejercicio $2 b$

Partiendo de la Figura 4 y 5 , se puede inferir que los estudiantes no tienen la habilidad de hallar el límite de una función por partes porque no saben aplicar las restricciones del dominio en cada función dada, esto les impide evaluar la función en cualquiera de las tres representaciones semióticas. Lo anterior argumenta lo presentado en la Tabla 6 , la cual nos muestra que el $44 \%$ de los estudiantes tuvieron un nivel de desempeño bajo.

El tercer ejercicio tenía como objetivo reconocer si lo estudiantes podían deducir una función por partes a partir de su gráfica representada en la figura 6 , además de determinar los límites marcados a través de los puntos A, B, D y E, es decir pasar de la representación gráfica a la tabular y algebraica.

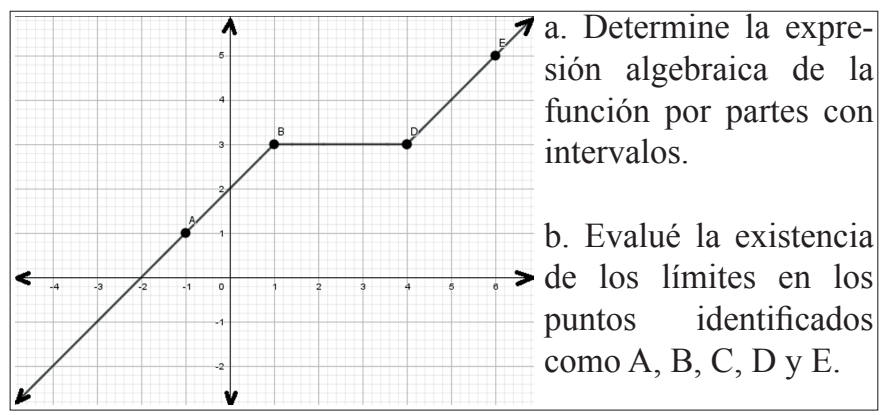

Las figuras 6 y 7 mostradas a continuación demuestran una aprehensión en el concepto de límite ya que pueden pasar de una representación semiótica a otra sin ningún problema, además de lograr determinar la función(es) representadas en la gráfica. Lo anterior se puede evidenciar en la Tabla 3 donde se muestra que el $67 \%$ de los estudiantes que eligieron la pregunta poseen un nivel alto en la resolución de este tipo de problemas.

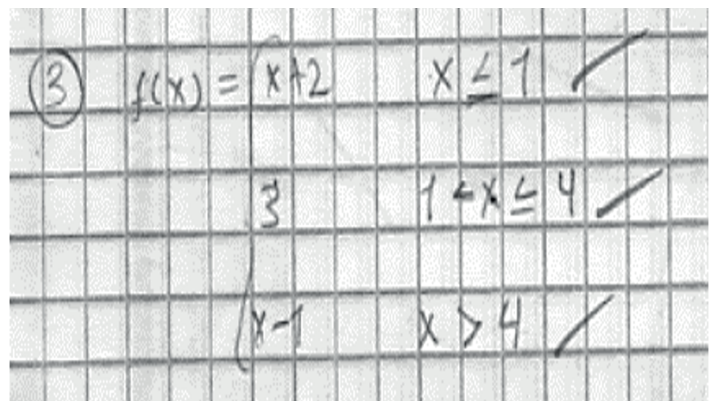

Figura 6. Resolución de ejercicio 3a

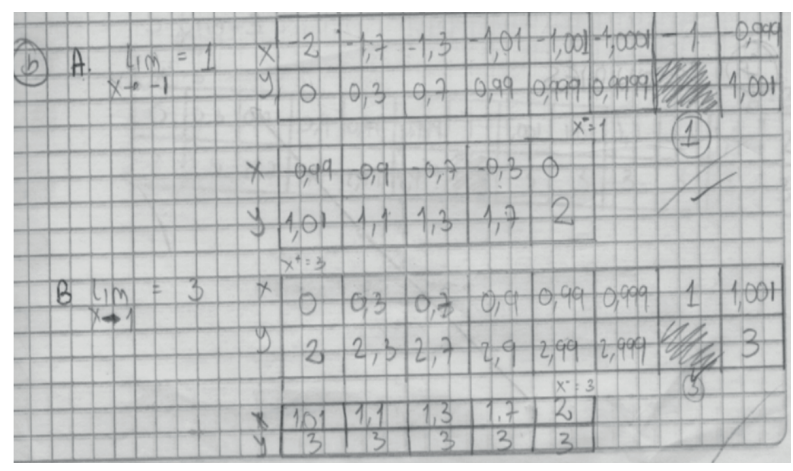

Figura 7. Resolución de ejercicio 3b (A y B)

El cuarto ejercicio tenía como objetivo reconocer si los estudiantes tenían la capacidad de utilizar el lenguaje natural para definir los conceptos de asíntota vertical, horizontal, no existencia de límite y límites que tienden al infinito.

4. Exprese con sus propias palabras ¿Qué es una asíntota vertical? y ¿Qué es una asíntota horizontal? y ¿Cuál es su relación con los conceptos de no existencia de límites o de límites que tienden al infinito? 
La Figura nos muestra que los estudiantes tienen noción de los conceptos, pero no logran expresarse con claridad, demostrando el no manejo del lenguaje natural matemático, por tal motivo el $88 \%$ de los estudiantes que seleccionaron esta pregunta tuvieron un nivel de desempeño medio, es decir no lograron completamente el objetivo planteado.

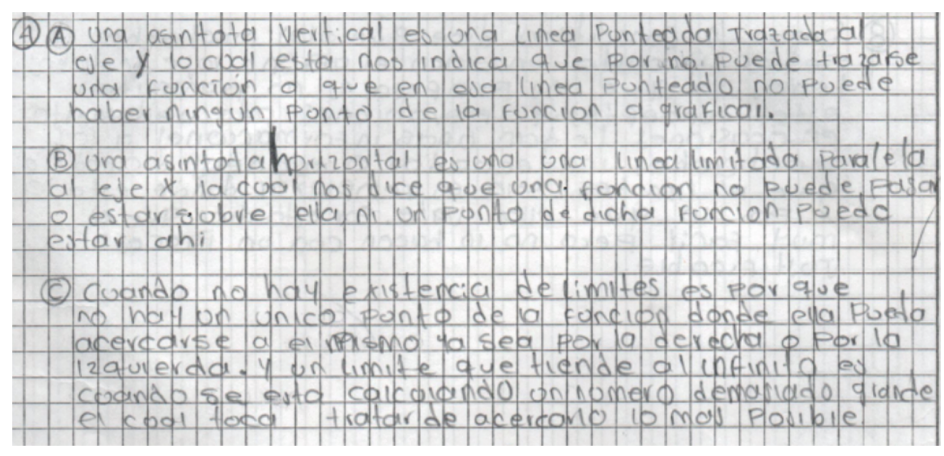

Figura 8. Resolución de ejercicio 4

El quinto ejercicio tenía como objetivo identificar si los estudiantes sabían calcular el límite de manera algebraica y utilizar propiedades para hallar los mismos.

\section{Determine la existencia del límite en cada caso:}
a. $\lim _{x \rightarrow \infty}\left(\frac{3 x}{\sqrt{x^{2}+5}}\right)$
b. $\lim _{x \rightarrow 1} \frac{x^{2}+x-2}{x^{2}-1}$

En la figura 9 se muestra que los estudiantes reconocen cuando un límite es indeterminado, pero no hacen uso de las diferentes propiedades existentes para calcular el límite, además del no manejo de los casos de factorización. Lo anterior demuestra las debilidades que tienen los estudiantes para determinar los límites a través de la representación algebraica, a pesar de que esta es la representación tradicional usualmente utilizada en el aprendizaje de las matemáticas, asimismo, demuestra lo obtenido en la Tabla 3 en donde se decía que el 54\% de los estudiantes se encontraban en un nivel de desempeño bajo.

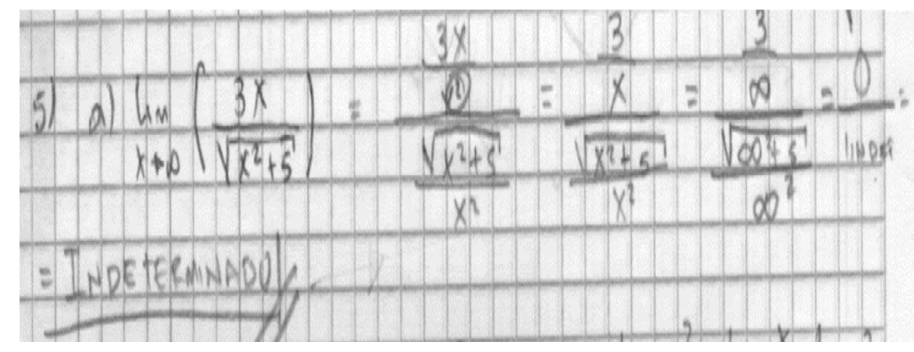

Figura 9. Resolución de ejercicio 5a

El sexto ejercicio tenía como objetivo reconocer si los estudiantes comprenden y aplican los conceptos de discontinuidad no evitable, asíntota y función creciente, asimismo que tengan la capacidad de representarlos de manera gráfica.
6. Construir una gráfica que satisfaga las siguientes condiciones: a. discontinuidad no evitable en $x=3$; b. con asíntota horizontal en $y=-2$; c. pasa por los puntos $(2,0)$ y $(-1,-1)$; d. creciente en el intervalo $(-\infty, 3)$.

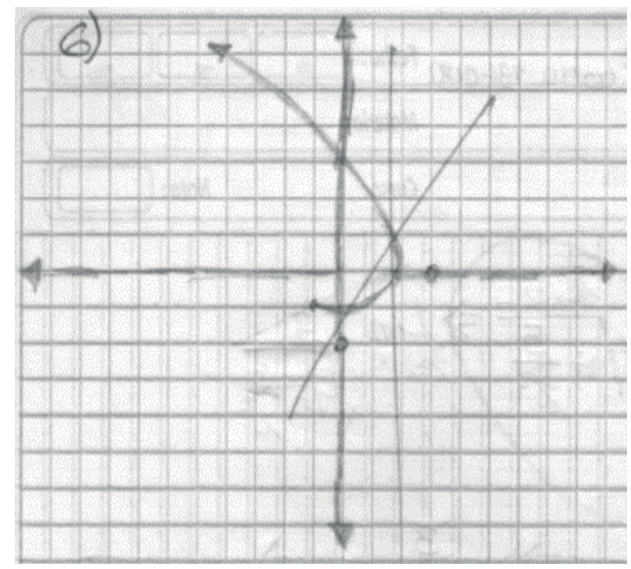

Figura 10. Resolución de ejercicio 6.

Al observar la Figura 10 se evidencia las falencias de los estudiantes al aplicar los conceptos mencionados anteriormente, a pesar de que en el ejercicio 1 hubo un buen nivel de desempeño en la comprensión del concepto de límite, esto no fue suficiente para que los estudiantes lograran el objetivo del ejercicio 6 , al contrario, el 55\% de los estudiantes obtuvieron un nivel de desempeño bajo.

El séptimo ejercicio tenía como objetivo reconocer si los estudiantes comprendían los conceptos de discontinuidad removible y no removible, a través de la representación gráfica $\mathrm{o}$ algebraica.

\section{Utilizando los registros gráfico y algebraico, represente las discontinuidades removibles y no removible.}

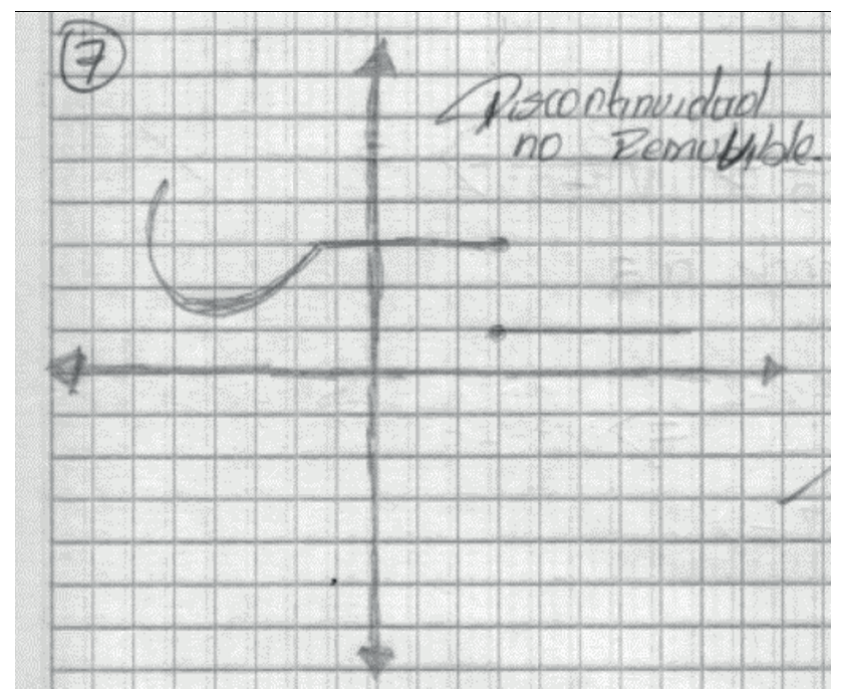

Figura 11. Resolución de ejercicio 7 (primera parte). 


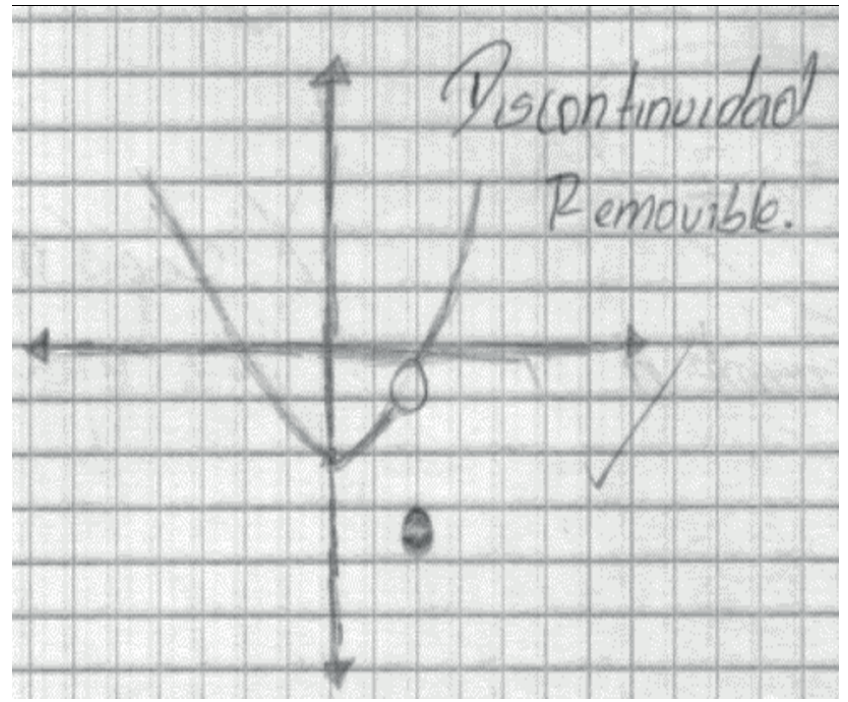

Figura 12. Resolución de ejercicio 7 (segunda parte).

La Tabla 6 muestra que el $83 \%$ de los estudiantes que eligieron el ejercicio 7 tuvieron un nivel de desempeño alto, es decir alcanzaron los obtuvieron planteados, esto se puede observar en las Figuras 11 y 12 en donde se representó el concepto de discontinuidad removible y no removible a través de gráficas.

\section{Conclusiones}

La enseñanza del concepto de límite es comúnmente abordada a través de la representación algebraica, dejando de lado los diferentes registros que se puede utilizar para tener mayor comprensión del concepto. Lo anterior ha ocasionado lagunas cognitivas por parte de los estudiantes, los cuales no tienen la capacidad de comprender el concepto de manera adecuada ya que solo conocen una manera de representarla.

Con base en lo anterior, se expone la importancia que tuvo la aplicación de las secuencias didácticas para fortalecer y desarrollar los conocimientos de los estudiantes alrededor del concepto de límite, a través de las diferentes representaciones semióticas (lenguaje natural, grafico, tabular y algebraico).

Los resultados muestran que el uso de diferentes representaciones para la resolución de problemas permite un mejor desempeño, lo anterior se evidencia en los ejercicios 1,3 y 7 en donde, más del $65 \%$ de los estudiantes lograron tener un nivel de desempeño alto. En cambio, aquellos ejercicios en donde se utilizaba un solo registro, se evidencio que más del $80 \%$ de los estudiantes se encuentran entre el nivel de desempeño bajo y medio.

Por otro lado, se destacan los resultados obtenidos en el ejercicio 1, dado que el $77 \%$ de los estudiantes lograron representar y argumentar situaciones en donde se debía utilizar el concepto de límite, asimismo los resultados del ejercicio 7 en donde el $83 \%$ logro representar el concepto de discontinuidad

\section{removible y no removible.}

Finalmente, los resultados de la aplicación de la pruebe permitirán al investigador reflexionar sobre ¿Cuáles son los cambios que se deben hacer en las secuencias didácticas para lograr que los estudiantes tengan un nivel de desempeño alto en el uso de cualquier representación semiótica? Se podría decir que la representación mayor dificultad fue la algebraica por lo que se recomienda reforzar el uso de este registro.

\section{Referencias}

Artigue, M., Douady, R., \& Moreno, L. (1995). Ingeniería didáctica en educación matemática. Una empresa docente ${ }^{\circledR} \&$ grupo editorial iberoamérica, s.a. de c.v., 148.

Blázquez, Ortega, Gatica, \& Banegas. (2006). Una conceptualización de límite para el aprendizaje inicial de análisis matemático en la universidad. Scielo.

Buendía Abalos, \& Molfino Vigo. (2010). El límite de funciones en la escuela: un análisis de su institulización. Revista electrónica de investigación en educación en ciencias.

Bustos, J., Naranjo, Y., Pisco, R., Torrez, G., \& Romero, I. (2016). Idea intuitiva de limite de una función en un punto. En diseño, implementación y evaluación de unidades didácticas de matemáticas en mad 2 (págs. 141-199). Bogotá.

Contreras de la Fuente, A., García Armenteros, M., \& Font Moll, V. (2012). Análisis de un proceso de estudio sobre la enseñanza del límite de una función. Scielo.

Donmez, \& Basturk. (2010). El conocimiento de los maestros de matemáticas antes del servicio de los diferentes métodos de enseñanza del concepto de límite y continuidad. Sciencedirect.

Engler, A., Gregorini, M., Vrancken, S., Müller, D., Hecklein, M., \& Henzenn, N. (2008). El límite infinito: una situación didáctica. Revista premisa.

Espíritu Montiel, V., \& Navarro, C. (2015). Límites indeterminados mediante el uso de tablas de valores y gráficas. Números. Revista de didactica de las matematicas, 31-53.

Medina M., A. (2001). Concepciones históricas asociadas al concepto de límite e implicaciones didácticas. Red academica, 19.

Morales Carballo, A., Reyes García, L., \& Hernández Gómez, J. (2013). El límite al infinito. Análisis preliminar para la elaboración de una estrategia metodológica de su enseñanza-aprendizaje. Revista premisa.

Navarro Sandoval, C., Romero Valencia, J., \& Miranda Nava, J. (2012). Una ingeniería didáctica para contribuir en la comprensión de la noción de límite en el nivel medio superior. En capítulo 2 propuestas para la enseñanza de las matemáticas (págs. 645-652). Mexico: comité latinoamericano de matemática educativa a. C.

Quevedo, J. M. (octubre de 2018). Un análisis de las concepciones acerca de las dificultades, los obstáculos y los errores relativos al límite. Tesis para optar el grado académico 
de magister en enseñanza de las matemáticas. Pontificia universidad católica del perú. Escuela de posgrado. Perú.

Radillo Enríquez, M., \& González Rendón, L. (2014). Enseñanza del concepto de límite de una función mediante sus diversas representaciones semióticas, a nivel licenciatura. Universidad de guadalajara, méxico.: comité latinoamericano de matemática educativa a. C.

Sanchez Gómez, C., \& Contreras De La Fuente, A. (1998). Analisis de manuales a través del tratamiento didáctico dado al concepto de limite de una función: una perspectiva desde la noción de obstáculo. Enseñanza de las ciencias, 73-84.

Sierra Vázquez, M., González Astudillo, M., \& López Esteban, C. (2000). Concepciones de los alumnos de bachillerato y curso de orientación universitaria sobre límite funcional y continuidad. Revista latinoamericana de investigacion en matematica educativa, 71-85.

Volveras Espinosa, A. (2015). Propuesta didactica para la enseñanza de limites de funciones en el grado undecimo de la i.e el rosario integrando geogebra. Universidad nacional de colombia sede manizales. Manizales.

Vrancken, Gregorini, Engler, Muller, \& Hecklein. (2006). Dificultades relacionadas con la enseñanza y el aprendizaje del concepto de límite. Articulos revista premisa. 